\title{
In vivo detection of endothelium dependent and independent pulmonary artery relaxation in children
}

David S Celermajer, Seamus Cullen, John E Deanfield

\begin{abstract}
Background-In vitro studies have suggested an important role for the endothelium in the control of pulmonary vascular tone, but endothelium dependent and independent relaxation of pulmonary arteries have not been studied in children in vivo.

Methods-The response of the pulmonary circulation to graded infusions of acetylcholine (an endothelial dependent vasodilator) and to nitroprusside (a dilator not dependent on endothelium) was studied in 10 children aged four to 16 years who had normal pulmonary haemodynamics. Arterial diameter was measured by quantitative angiography, and pulmonary blood flow velocity was measured with a 3F intra-arterial Doppler catheter placed in a lower lobe segmental artery.
\end{abstract}

Results-There was a dose dependent increase in flow velocity in response to acetylcholine (maximum response 93\%) (SEM 7\%), and an increase of $51 \%(8 \%)$ in response to nitroprusside. By contrast, segmental artery diameter was unchanged during acetylcholine infusion in all patients, and increased only modestly in response to nitroprusside (5\% (1\%)).

Conclusions-The most important site of action of endothelium dependent and independent pulmonary vasodilators is distal to the segmental pulmonary arteries. Despite low resting tone in the pulmonary circulation, endothelium dependent vasodilatation can be shown in vivo. This may allow study of the role of endothelial dysfunction in children with abnormal pulmonary haemodynamics secondary to congenital heart disease.

(Br Heart f 1993;69:298-302)

Cardiothoracic Unit, Hospitals for Sick Children, Great Ormond Street, London

D S Celermajer

$S$ Cullen

J E Deanfield

Correspondence to:

Dr D S Celermajer,

Cardiothoracic Unit

Hospitals for Sick Children,

Great Ormond Street,
London WCIN $3 \mathrm{JH}$.

Accepted for publication

12 October 1992.
Healthy vascular endothelium is known to produce a relaxing factor that exerts important control over local vasomotor tone,,$^{12}$ and that mediates the vasorelaxant action of endogenous vasodilators such as acetylcholine. In lambs, endothelium derived relaxing factor in part mediates the low resistance of the pulmonary circulation. ${ }^{34}$ Study of human pulmonary arteries in vitro also suggests that endothelium maintains low resistance by secretion of relaxing factor (or factors), ${ }^{5}$ but such arteries may still dilate in response to acetylcholine or adenosine diphosphate. ${ }^{67}$ Despite this, apart from two preliminary reports on adult subjects, ${ }^{89}$ in vivo data on the role of pulmonary endothelium in modulating vascular tone in human subjects are lacking.

A potential role for endothelial dysfunction as an early event in the pathophysiology of pulmonary vascular disease has been postulated, ${ }^{10}$ and structural abnormalities of endothelial cells have been shown in the first years of life in children with congenital heart $i$ disease and pulmonary hypertension. ${ }^{11}$ An in $\infty$ vivo test of pulmonary endothelial function would permit study of the role of endothelial dysfunction in the pathogenesis of pulmonary hypertension in various clinical settings. We therefore studied the response of the pulmonary circulation to endothelium dependent and independent vasodilators in children with normal pulmonary haemodynamics.

\section{Patients and methods}

PATIENTS

As normal children do not undergo cardiac catheterisation, we selected for study 10 children aged four to 16 years with congenital heart disease that did not alter their pulmonary haemodynamics. Six children had mild left heart obstructive lesions, such as aortic stenosis or coarctation, three had tiny intracardiac defects with no detectable left to right shunt, and one child had a fistula from the left coronary artery to the left atrium. These patients had each had echocardiography, and underwent cardiac catheterisation as part of their further management, at the discretion of their consultant cardiologist. In all 10 patients the mean pulmonary artery pressure was $\leqslant 15 \mathrm{~mm} \mathrm{Hg}$, the left ventricular end diastolic pressure was $\leqslant 8 \mathrm{~mm} \mathrm{Hg}$, and the pulmonary resistance was $\leqslant 1.5$ units. $\mathrm{m}^{2}$ (calculated with oxygen saturations, assumed oxygen consumption, and the Fick principle). None had had any previous surgery, and none were taking cardioactive medications. The study protocol was approved by the institution's committee on ethical practice, and informed written consent was obtained from the parents of all patients.

\section{STUDY DESIGN}

Diagnostic cardiac catheterisation was performed under local anaesthesia and benzodiazepine sedation through a percutaneous femoral approach, and haemodynamic data were taken. Heparin (50 units $/ \mathrm{kg})$ and 
Figure 1 Position of the Doppler catheter for flow velocity measurement and the catheter for angiography in the pulmonary artery. The segment analysed by quantitative angiography is indicated by the stippled box. ECG, electrocardiogram; $P A P$ pulmonary artery pressure.

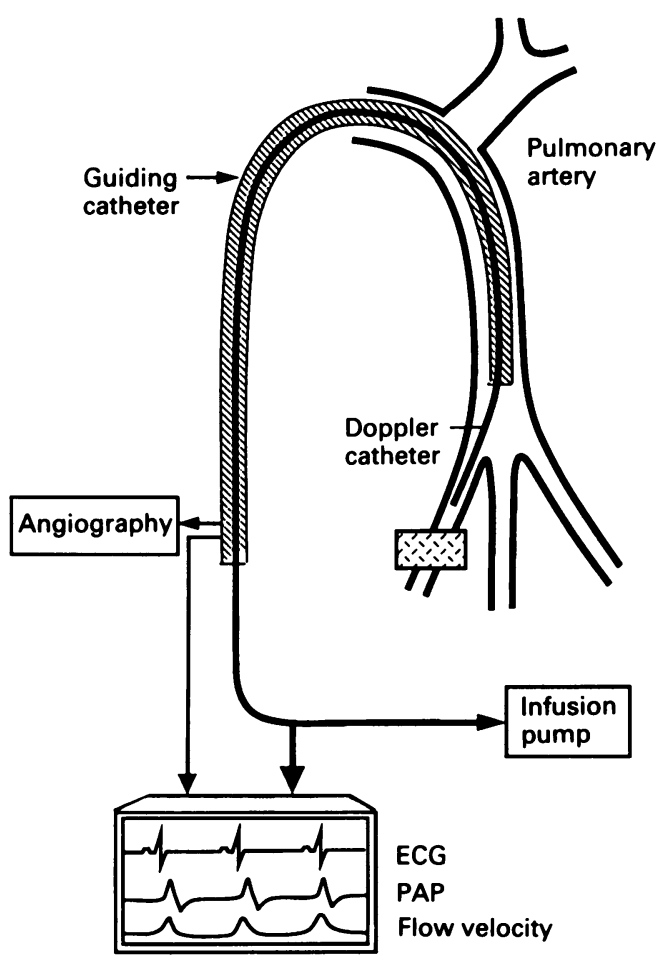

diazepam $(0 \cdot 1-0.3 \mathrm{mg} / \mathrm{kg})$ were given intravenously before the vascular study began. Studies were performed before diagnostic angiography. Arterial blood gases were taken at the beginning, during, and at the end of each study to exclude carbon dioxide retention and to document respiratory stability throughout the infusion. Dependent on patient size, a 5,6 , or $7 F$ long biopsy sheath (Cordis, UK and Cook, UK) was inserted into the left or right lower lobe pulmonary artery. A $20 \mathrm{MHz}$ pulsed Doppler crystal side mounted on a $3 F$ catheter (Wessex Medical, Midhurst, England) was positioned through the biopsy sheath into a straight segment of the medial or posterior branch of the lower lobe pulmonary artery. If neither of these branches had a straight segment, the Doppler catheter was positioned in the distal lower lobe artery (fig 1). Position in the centre of this vessel was confirmed by "sighting" angiography and a stable flow velocity signal with minimal noise. The Doppler catheter was connected through a flow velocimeter box (Millar Instruments Inc, Houston, Texas) to a multichanel recorder, that dis-

Figure 2 The infusion protocol for the pulmonary artery studies. $\mathrm{C1}$ and $\mathrm{C2}$ control infusions; $A C h$,

7 , and 6, acetylcholine

infusions of $10^{-8}, 10^{-7}$, and $10^{-6} \mathrm{M} ; \mathrm{NP}$, nitroprusside. played the electrocardiogram, pulmonary arterial pressure, and the mean or phasic Doppler flow velocity.

Serial infusions with an infusion pump (Harvard Apparatus, Edenbridge, UK) were made at a rate of $0.8 \mathrm{ml} / \mathrm{min}$ through the Doppler catheter into the segmental pulmonary artery in the sequence: (a) a four minute control infusion ( $5 \%$ dextrose); (b) three acetylcholine infusions each lasting four minutes, with a final estimated concentration of $10^{-8}, 10^{-7}$ and $10^{-6} \mathrm{M} ;(c)$ an eight minute repeat control infusion; and (d) a four minute infusion of sodium nitroprusside at $0 \cdot 1$ $\mu \mathrm{g} / \mathrm{kg} / \mathrm{min}$ (fig 2). If the systemic arterial pressure was stable, the nitroprusside infusion was continued at double the given rate for a further two minutes. Throughout the protocol the heart rate, systemic and pulmonary arterial pressure, and the electrocardiogram were monitored continuously. Thirty seconds before the end of each infusion Doppler flow velocities were recorded. The values for mean and phasic flow velocity were calculated automatically, and read directly from the chart recorder. Flow velocity during each infusion was expressed as a percentage relative to the velocity during the first control infusion.

\section{QUANTITATIVE ANGIOGRAPHY}

At the end of each infusion, a single plane digital subtraction angiogram (Digitron 2 system, Siemens) was performed in the anteroposterior projection, or a few degrees of left of anterior oblique if the Doppler catheter overlay the left cardiac border. Contrast was injected through the long biopsy sheath: one third strength non-ionic contrast material (Ultravist, by Schering, UK) made up in normal saline was given; $0.3-0.5 \mathrm{ml} / \mathrm{kg}$ at a rate of $0.3-0.5 \mathrm{ml} / \mathrm{kg} / \mathrm{s}$ through a power injector (Angiomat by Phillips, UK), to optimise the quality and reproducibility of the opacification. After each angiogram (biplane system by Siemens, UK), fluoroscopy was performed in anteroposterior and lateral projections to confirm the stability of the Doppler catheter position.

For each angiogram, the first four diastolic frames with good, even opacification of the lower lobe artery were selected. Analysis was performed by an observer blinded to the patient's diagnosis. Each frame was magnified 16 times, and the diameter of a segment of straight artery, just below the tip of the Doppler catheter, was measured. The same arterial segment was analysed for each infusion and a computerised measurement of diameter was calculated with the "per cent stenosis" software package (Siemens). Arterial diameter was calculated in pixels on a $512 \times 512$ matrix and expressed as percentage relative to the diameter of the vessel in the first control infusion.

\section{STATISTICAL ANALYSIS}

All data are expressed as mean (SEM). Results for each experimental condition were expressed as a percentage relative to the first control value. The Student's $t$ test was used

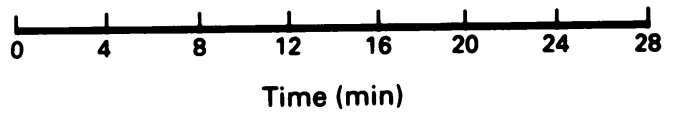




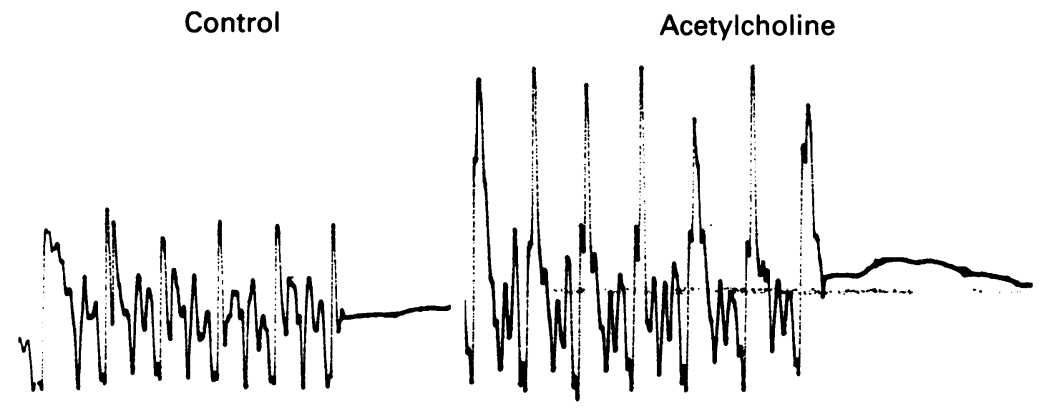

Figure 3 Phasic and mean flow velocity traces from an 11 year old boy during control and acetylcholine infusions show considerable increase in pulmonary flow velocity with acetylcholine. occurred in response to acetylcholine; during infusions of $10^{-8}, 10^{-7}$, and $10^{-6} \mathrm{M}$ acetylcholine, the flow velocity increased by $16 \%(7 \%), 60 \%(10 \%)$, and $93 \%(7 \%)$ (range $51 \%-131 \%, \mathrm{p}<0.01$ compared with baseline value). After the second control infusion, flow velocity returned to its baseline value $(9 \%(4 \%), N S)$. Flow velocity also increased in response to nitroprusside infusion ( $51 \%(8 \%)$, range $10 \%-100 \%, p<0.01$ compared with baseline) (figs 3-5).

\section{ARTERIAL DIAMETER}

The diameter of the segments analysed varied from $2 \cdot 1$ to $10.5 \mathrm{~mm}(5 \cdot 3(0 \cdot 8) \mathrm{mm})$. The diameter did not change significantly during any of the acetylcholine infusions; after $10^{-6}$ $M$, the diameter was increased by $1 \%(1 \%)$ (range 2 to 7 ) compared with the baseline value (NS). During nitroprusside infusion there was a small but significant increase in diameter $(5 \%(1 \%)$, range 1 to $12, \mathrm{p}<0.05$ compared with baseline). There was no significant relation between the size of the vessel measured and the increase in flow velocity to acetylcholine $(r=0.40)$ or nitroprusside $(r=$ $0 \cdot 43)$.

Ten (five boys, five girls) children aged to 16 (mean 9 (1)) years were studied. Pulmonary to systemic flow ratio was 1.0 in all, mean pulmonary artery pressure was 11(1) $\mathrm{mm} \mathrm{Hg}$, and pulmonary resistance index was $1.0(0 \cdot 1)$ units.m..$^{2}$ Mean systemic arterial pressure was $65(4) \mathrm{mm} \mathrm{Hg}$.

\section{HAEMODYNAMIC AND RESPIRATORY MONITORING}

In nine of 10 patients, the heart rate, arterial blood gas measurements, and systemic arterial and pulmonary arterial pressures remained constant throughout the infusion. In one control patient all variables were stable until the third minute of the nitroprusside infusion, at which time systemic hypotension and tachycardia were noted; these values returned promptly to normal when infusion of the drug was stopped. Therefore analysis of response to nitroprusside was based on only nine patients. All patients tolerated the procedure well.

\section{FLOW VELOCITY}

A dose dependent increase in flow velocity

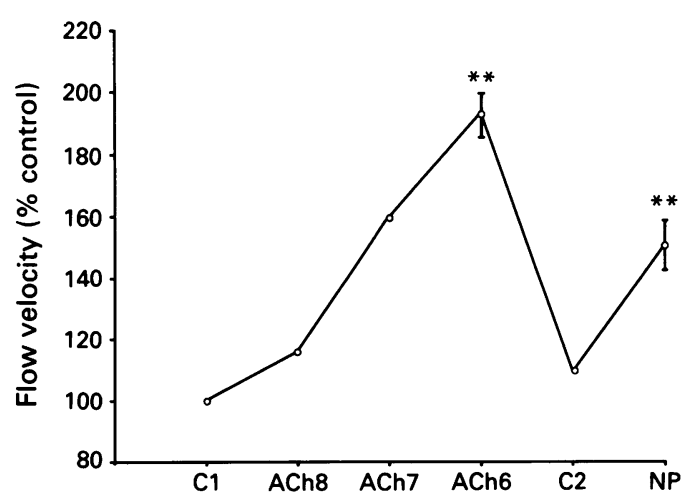

Figure 4 Changes in pulmonary arterial flow velocity in response to three doses of acetylcholine ( $A C h$ 8, 7, and 6 equal to estimated local concentrations of $10^{8}, 10^{-7}$, and $\left.10^{-6} \mathrm{M}\right)$ and to nitroprusside $(N P) .{ }^{\star \star_{p}}<0.01$.

\section{Discussion}

This study shows that endothelium dependent and independent vasodilatation occur in the lungs of children with normal pulmonary haemodynamics. Given that there was almost no change in segmental artery diameter in response to acetylcholine but a large increase in flow velocity, the most important site of endothelial dependent pulmonary artery relaxation must be distal to the large arteries. Preliminary reports from two groups studying pulmonary haemodynamics in adults also found that diameter of the conduit pulmonary arteries did not change in response to

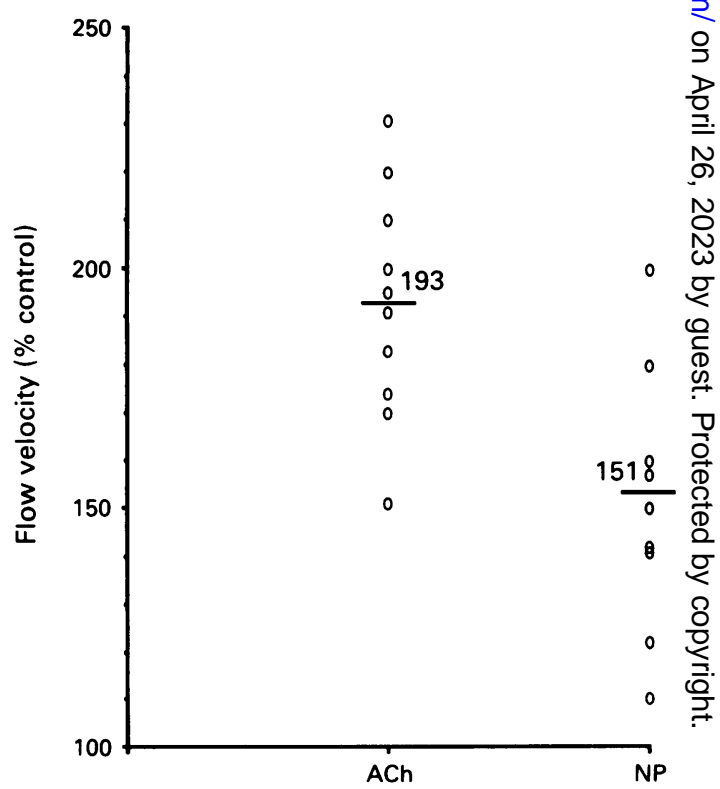

Figure 5 Maximal increase in flow velocity in pulmonary arteries of each patient in response to acetylcholine (ACh) and nitroprusside (NP). Horizontal lines indicate the mean value. 
infusions of acetylcholine or nitroprusside. ${ }^{10} 11$ The lack of substantial increase in the diameter of a large artery after vasodilator infusions in vivo compared with in vitro studies on human pulmonary artery rings ${ }^{6} 12$ is probably because in vitro investigators have preconstricted the vessels to elicit a vasodilator response.

This in vivo method for the study of endothelial function is similar to that described by several groups to study the coronary circulation. ${ }^{21314}$ All patients were catheterised under local anaesthetic to avoid any potential influence of anaesthetic agents on pulmonary endothelial function, ${ }^{15}$ and long infusion times were chosen to ensure that any transient effect of contrast material on the endothelium would have worn off; ${ }^{16}$ use of diluted contrast at normal osmolarity minimised these effects, and in our pilot studies, injection of contrast caused a reversible rise in flow velocity that returned to baseline values within 60 seconds. The use of a $3 F$ Doppler intra-arterial catheter has previously been shown to be non-obstructive to flow, safe, and reliable. ${ }^{17}$ Doppler catheters have been validated for measurement of absolute flow velocity, but at low flow rates may underestimate flow. ${ }^{18}$ Side mounted Doppler catheters are reliable for the measurement of flow ratios and relative changes of flow velocity. ${ }^{17-19}$ Quantitative angiography is a well validated technique for measurement of arterial diameter, and in vessels $>1 \mathrm{~mm}$ in diameter provides accurate and reproducible results. ${ }^{20-22}$ In this study the 10 children were of different ages and sizes, and therefore we have used each child as their own control and compared each observation with the first baseline result for each child. Calculation of vascular resistance was based on assumed rather than measured oxygen consumption; the potential error thereby introduced was likely to be small in these patients with otherwise normal pulmonary haemodynamics.

In 1957 Fritts et al reported that an infusion of acetylcholine into the lungs of normal human subjects produced a fall in pulmonary arterial pressure, and that this effect was exaggerated if the pulmonary circulation was preconstricted by hypoxia. ${ }^{23}$ In the intact lungs of cats and rabbits, acetylcholine produces vasoconstriction; only in preconstricted arteries does acetylcholine induce vasodilatation. ${ }^{24-26}$ Numerous studies on intact humans, however, have shown that acetylcholine lowers pulmonary pressure and vascular resistance; $;^{23-29}$ this response is now thought to be due to the release of relaxing factors from the endothelium that act on underlying smooth muscle in vessels with basal vasoconstrictor tone. This hypothesis has been supported by in vitro studies, ${ }^{7}$ and was confirmed by our findings in children.

The maximum increases induced in pulmonary blood flow in our children (about $90 \%$ with acetylcholine and $50 \%$ with nitroprusside) are similar to the maximum relaxation that has been found in response to these agents in isolated pulmonary arteries from normal subjects studied in vitro (about $80 \%$ and $40 \%){ }^{7}$ Both in vivo and in vitro, vasodilatation was greater in response to acetylcholine than to nitroprusside. This may be because nitroprusside is a relatively weak stimulator of pulmonary vascular smooth muscle compared with endothelium dependent relaxing factor, the endogenous nitrovasodilator, or that (at least in vivo) the dose schedule elicited the maximal responses to acetylcholine, but a submaximal response to nitroprusside; for nitroprusside the dose range is limited by its powerful systemic vasodilator effect.

The availability of an in vivo test of endothelial function in children may permit further study of the role of endothelial dysfunction in the pathogenesis of pulmonary vascular disease. We are therefore currently studying endothelial function in children with abnormal haemodynamics secondary to congenital heart disease.

This work is supported by a grant from the British Heart Foundation. We thank Mary Jane Potter for her secretarial assistance.

1 Furchgott RF, Zawadzki JV. The obligatory role of endothelial cells in the relaxation of arterial smooth muscle by acetylcholine. Nature 1980;288:373-6.

2 Ludmer PL, Selwyn AP, Shook TL, Wayne RR, Mudge $\mathrm{GH}$, Alexander RW. Paradoxical vasoconstriction induced by acetylcholine in atherosclerotic coronary arteries. $N$ Engl f Med 1986;315:1046-51.

3 Fineman JR, Heymann MA, Soifer SJ. N-nitro-L-arginine attenuates endothelium-dependent pulmonary vasodilaattenuates endothelium-dependent pulmonary vas

4 Fineman JR, Chang R, Soifer SJ. L-arginine, a precursor of EDRF in vitro, produces pulmonary vasodilation in lambs. Am f Physiol 1991;261:H1563-9.

5 Cremona G, Dinh-Xuan AT, Higenbottam TW. Endothelium-derived relaxing factor and the pulmonary circulation. Lung 1991;169:185-202.

6 Dinh-Xuan AT, Higenbottam TW, Pepke-Zaba J, Wells FC, Wallwork J. Acetylcholine and adenosine diphosphate cause endothelium-dependent relaxation of isolated human pulmonary arteries. Eur Respir ff 1990; 3:633-8.

7 Dinh-Xuan AT, Higenbottam TW, Clelland CA, et al. Impairment of endothelium-dependent relaxation in chronic obstructive lung disease. $N$ Engl $₹$ Med 1991; 324:1539-47.

8 Landzberg MJ, Vita JA, Yeung AC, Cannon CP, Ganz P, Selwyn AP. Endothelium-dependent modulation of pulmonary vascular tone in man [abstract]. $\mathcal{F} \mathrm{Am}$ Coll Cardiol 1992;19(suppl A):211A.

9 Porter T, Taylor D, Cycan A, Bagley C, Moharty P, McGuire VA. Impaired endothelium-dependent pulmonary arterial responses in heart failure: influence of pulmonary hypertension [abstract]. $\mathcal{F} \mathrm{Am}$ Coll Cardiol 1992;19(suppl A):2A.

10 Rich S, Brundage BH. Pulmonary hypertension: a cellular basis for understanding the pathophysiology and treatment. F Am Coll Cardiol 1989;11:545-50.

11 Hall SM, Haworth SG. Onset and evolution of pulmonary vascular disease in young children: abnormal postnatal remodelling studied in lung biopsies. $₹$ Pathol 1992; 166:183-94

12 Greenberg B, Rhoden K, Barnes PJ. Endothelium-dependent relaxation of human pulmonary arteries. $A m f$ Physiol 1987;252:H434-8.

13 Nabel EG, Selwyn AP, Ganz P. Large coronary arteries in humans are responsive to changing blood flow: an endothelium dependent mechanism that fails in patients with atherosclerosis. $\mathcal{f} \mathrm{Am}$ Coll Cardiol 1990;16:349-56.

14 Vita JA, Treasure CB, Nabel EG, et al. Coronary vasomotor responses to acetylcholine relates to risk factors for coronary artery disease. Circulation 1990;81:491-7.

15 Robinson S. Anesthesia for congenital heart disease. In Gregory G, ed. Pediatric anesthesia. New York Churchill Livingstone, 1983:607-41.

16 Lange PE, Neubert D, Onnasch DGW, Sievers HH, Heintzen PH. Effects of angiographic contrast media on the pulmonary circulation in pigs. $A m$ f Cardiol the pulmonary circ.

17 Sibley DH, Millar HD, Hartley CJ, Whitlow PL. Subselective measurement of coronary flow velocity Subselective measurement of coronary flow velocity using a steerable

18 Hangiandreou NJ, Toggart EJ, Mistretta CA. Investigation of the performance of two types of the 
Doppler catheter in vitro. Cathet Cardiovasc Diagn 1989; 18:108-17.

19 Hartley CJ. Review of intracoronary Doppler catheters. Int $\mathcal{f}$ Card Imaging 1989;4:159-68.

20 Mancia GBJ, Simon SB, McGillem MJ, LeFree MT, Friedman HZ, Vogel RA. Automated quantitative arteriography: morphologic and physiologic validation in vivo of a rapid digital angiographic method. Circulation 1987;75:452-60.

21 Spears JR, Sandor T, Als AV, et al. Computerised image analysis for quantitative measurement of vessel diameter for cineangiograms. Circulation 1983;68:453-61.

22 Katritsis D, Webb-Peploe MM. The role of digital subtraction angiography in the quantitation of coronary stenosis. Int $f$ Cardiol 1990;29:277-83.

23 Fritts HW Jr, Harris P, Clauss RH, Odell JE, Cournand A. The effect of acetylcholine on the human pulmonary circulation under normal and hypoxic conditions. $\mathcal{f}$ Clin circulation under normal
Invest 1958;37:99-110

24 Hyman AL, Kadowitz PJ. Differences in response to acetylcholine in the cat and rabbit pulmonary vascular bed [abstract]. Circulation 1987;76(suppl IV):233.

25 Nandiwada PA, Hyman AL, Kadowitz PJ. Pulmonary vasodilator responses to vagal stimulation and acetylvasodilator responses to vagal stimulation

26 Cherry PD, Gillis CN. Evidence for the role of endothelium-derived relaxing factor in acetylcholine-induced vasodilatation in the intact lung. $f$ Pharmacol Exp Ther 1987;241:516-20.

27 Oakley C, Glick G, Luria MN, Schreiner BF, Yu PN. Some regulatory mechanisms of the human pulmonary vascular bed. Circulation 1962;26:917.

28 Bishop JM, Harris P, Bateman M, Davidson LAG. The effect of acetylcholine upon respiratory gas exchange in mitral stenosis. $\mathcal{Y}$ Clin Invest 1961;40:105.

29 Bjure J, Helander E, Lindell S-E, Soderholm B, Westling H. Effect of acetylcholine and histamine on the pulmonary circulation in normal men and women. Scandinavian fournal of Respiratory Diseases 1967;48: 214. 